\title{
Potential of Polysorbate20 Surfactant for Enhanced Oil Recovery
}

\author{
Wan Rosli Wan Sulaiman*,1,2 Sang Kwon Park ${ }^{1}$ and Euy Soo Lee ${ }^{1}$ \\ ${ }^{I}$ Department of Chemical and Biochemical Engineering, Dongguk University, Seoul, Korea; ${ }^{2}$ Petroleum Engineering \\ Department, Faculty of Petroleum and Renewable Energy Engineering, Universiti Teknologi Malaysia, Malaysia
}

\begin{abstract}
Surfactant for enhanced oil recovery (EOR) has been applied for many years, particularly in the 1970's and 1980 's when the technology was put on a sound scientific basis. Unfortunately, the economic reality of the process performance in field trials has precluded widespread deployment of this technology. Many surfactants have been evaluated for their ability to recover incremental oil and this study is focusing on Polysorbate20 as a candidate for this EOR application. This laboratory study aims to determine the characteristics of Polysorbate20 surfactant, in particular for its capabilities to create low interfacial tensions (IFT) with n-alkane hydrocarbons. Certain formulated surfactant and cosolvent exhibit low interfacial tension (IFT) values of $0.01 \mathrm{dyne} / \mathrm{cm}$ or less versus n-octane. This surfactant was tested for EOR using coreflood tests on Berea sandstones. Laboratory tests had confirmed that the useful property which is to reduce the IFT by using Polysorbate20 formulations can be largely independent of both salinity and temperature. Preliminary studies also suggest Polysorbate20 has only modest adsorption between 0.10 to $0.11 \mathrm{mg} / \mathrm{g}$ onto crushed sandstone and between 15.33 to $17.62 \mathrm{mg} / \mathrm{g}$ onto kaolinite clay.
\end{abstract}

Keywords: Enhanced oil recovery, surfactant, Polysorbate20, interfacial tension, kaolinite clay.

\section{INTRODUCTION}

Surfactant flood for enhanced oil recovery (EOR) schemes has been employed for more than 35 years, particularly in the USA for depleted oil reservoirs after waterflooding $[1,2]$. Most of the field trials were of pilot scale but several commercial scale projects were also executed. Nowadays, because of the high oil price, this technology is experiencing increasing interest.

This study considers Polysorbate20, one type of surfactant largely ignored as candidates for EOR applications. Polysorbate20 is a nonionic surfactant and emulsifier derived from sorbitan monolaurate, and is distinguished from the other members in the polysorbate range by the length of the polyoxyethylene chain and the fatty acid ester moiety. It is used as a dispersing agent, mixes oil and water, fragrance solubilizer and stabilizer and has a soothing effect on the skin [3].

Polysorbate20 has gained favor as economical processes were developed to manufacture them on a large scale, and also because there has been an increased drive to use surfactants with favorable, low toxicity characteristics for many purposes. This surfactant now sees widespread use in household detergents, cosmetics, and agricultural products $[3,4]$.

During a waterflood process, the capillary number $\mathrm{N}_{\text {cap }}$ (Eq. 1) is around $10^{-8}$ to $10^{-7}$ and has to be increased by an order of at least two to three magnitudes [5] to enable additional oil recovery. The approach to increase oil

*Address correspondence to this author at the Department of Chemical and Biochemical Engineering, Dongguk University, 3-26, Pil-Dong, Chung-gu, Seoul, 100-715 Korea; Tel: +82-10-8320-2711; Fax: +82-2-2266-1848;

E-mail: r-wan@petroleum.utm.my production and $\mathrm{N}_{\text {cap }}$ is to significantly decrease the IFT to low levels (i.e. $0.01 \mathrm{mN} / \mathrm{m}$ ) by using specially formulated surfactant. The surfactant solution alters the wettability of the reservoir rock surfaces, making it more water wet. This process has the effects of allowing the wetting aqueous phase to imbibe into the rock matrix spontaneously and expel oil from the matrix.

$\mathrm{N}_{\text {cap }}=\mathrm{v} . \mu / \mathrm{IFT}$

where,

$$
\begin{array}{ll}
\mathrm{N}_{\text {cap }} & =\text { capillary number } \\
\mathrm{v} & =\text { Darcy velocity }(\mathrm{m} / \mathrm{s}) \\
\mu & =\text { Viscosity of injected phase (Pa.s) } \\
\mathrm{IFT} & =\text { Interfacial tension between oleic phase and aqueous } \\
& \text { phase }(\mathrm{N} / \mathrm{m})
\end{array}
$$

\subsection{Potential advantages of Polysorbate20}

The Polysorbate 20 has some interesting and potential useful properties as EOR agents:

1) When mixed with a hydrophobic co-surfactant (e.g. an alcohol or some other surfactants), a middle-phase micro emulsion may appear and in some cases it can create a low IFT (0.01 dyne/cm or less).

2) A remarkable property for Polysorbate 20 is to have a phase behavior and IFT which is independent of temperature and salinity. Surfactant formulations that create a low IFT irrespective of temperature and salinity would be a very useful property for oilfield EOR applications [6, 7]. Theoretical aspects also indicate that having these large head group and the nonionic molecule are consistent with the observation of the phase behavior 
and IFT being indifferent to changes in the temperature and the salinity.

3) This surfactant is already available as commercial product and already in use in significant quantities for other industrial applications [8].

4) Polysorbate20 is manufactured from renewable resources and therefore its cost is largely uncoupled from the current price of crude oil.

\section{EXPERIMENTAL METHODOLOGY}

From the basic screening analysis, which included IFT measurement and adsorption measurements, the best performing surfactant-cosolvent combination was selected for further test on coreflood experiment. Performance in term of EOR means that the surfactant can generate a low IFT and also shows low adsorption on reservoir rock material $[9,10]$.

\subsection{Materials}

The study focuses on the behavior of Polysorbate20 surfactant. The hydrophile-lipophile balance (HLB) value of this surfactant is 8.7 as given by the supplier and its structure is shown in Fig. (1). The HLB is a measure of the molecules ability in creating emulsions and is related to its oil/water solubility. Higher HLB indicates greater water solubility.

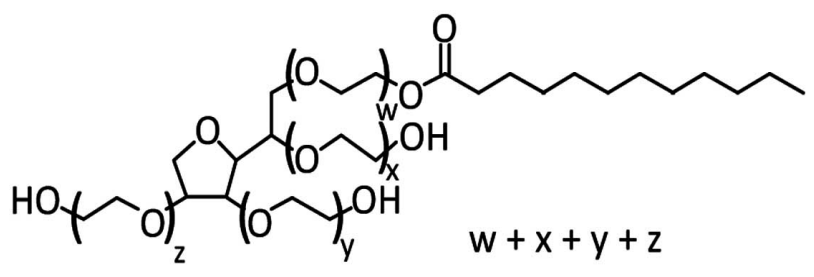

Fig. (1). Chemical structure of Polysorbate20.

For the hydrocarbon phase, n-octane was used as a model compound. Other studies have shown that IFT and phase behavior of crude oils often can be represented by n-alkanes ranging from n-hexane to n-decane. Thus, this current study has selected n-octane as a 'typical' representative hydrocarbon. In other words, surfactant formulations that are effective in reducing IFT versus n-octane are likely good candidates also for mobilizing crude oils.

The surfactant, n-octane, sodium chloride, 1-propanol, 1butanol, 1-hexanol, 1-octanol and kaolinite were purchased from Sigma Aldrich. The de-ionized water was prepared in house by distilling tap water twice. Berea core sandstone was purchased from Cleveland Quarries, USA. The properties of Berea core sandstone are described in the Table $\mathbf{1}$ below.

\subsection{Preparation of Surfactant-Cosolvent Formulations}

All formulations were prepared in $2 \mathrm{wt} \% \mathrm{NaCl}$ brine. The surfactant (concentration varies between $0.25-1.50 \mathrm{wt} \%$ ) was dissolved in the brine and the mixture was stirred for 30 minutes. Then the mixture was equilibrated for 24 hours. After that the mixture was split into four parts and added with different cosolvent (co solvent concentration is $1 \mathrm{wt} \%$ ). These formulations were stirred again for 30 minutes and put on the rest for another 24 hours to reach equilibration. After equilibration, the formulations were centrifuged at $2000 \mathrm{rpm}$ for 15 minutes to remove any excess of solid materials and only the supernatant was used for core flooding injection to avoid blocking of the pore network by solid particles. All steps occurred at room temperature and atmospheric pressure.

\section{Table 1. Properties of Berea Sandstone}

\begin{tabular}{|l|l|}
\hline Core properties & Value \\
\hline \hline Rock mass [g] & $1642 \mathrm{~g}$ \\
\hline Connected pore volume, PV [mL] & $161 \mathrm{~mL}$ \\
\hline Dead pore volume, $[\mathrm{mL}]$ & $32 \mathrm{~mL}$ \\
\hline Length, L [cm] & $30.48 \mathrm{~cm}$ \\
\hline Diameter, D [cm] & $6.35 \mathrm{~cm}$ \\
\hline Porosity, $\Phi$ & 0.20 \\
\hline Brine permeability [md] & $300 \mathrm{md}$ \\
\hline
\end{tabular}

\subsection{Phase Behavior}

Test tube samples were prepared with $5 \mathrm{ml}$ of aqueous formulations and $5 \mathrm{ml}$ of n-octane. After mixing well for several minutes, they were allowed to stand for a few days to allow the fluids to reach equilibrium phase at ambient conditions. The physical appearance of the phases was noted such as the relative volumes of the aqueous and oleic phases, and if any third, so-called middle-phase forms.

\subsection{Interfacial Tension (IFT)}

The IFT was determined for selected phase equilibrated test tube samples by using a spinning drop tensiometer (from Temco, Inc.). A glass tube was loaded with the aqueous phase followed by injection of a few micro-liters of the uppermost oleic phase. The glass tube was spun in the instrument at a know speed and the IFT determined from the oil drop geometry. Since the samples already come from fluids at phase equilibrium, typically it required less than one hour for the measured IFT to stabilize to a final value.

\subsection{Surfactant-Solid Adsorption}

Polysorbate20 surfactant adsorption onto kaolinite clay and crushed sandstone were measured. All of these tests were conducted at $25^{\circ} \mathrm{C}$ with a weight ratio of liquid to solid of 20, and for a mixing exposure period of 12 hours. Kaolinite was selected as the adsorbent of choice because it is among the most common clays found in oil reservoirs. Kaolinite is also a stable material which does not swell when immersed in water [11]. Adsorption of surfactant also measured on crushed sand stone.

During a surfactant coreflood, surfactant can fade by adsorption onto the porous medium [12]. Literature values for surfactant adsorption onto Berea core material range from 0.1 to $1.2 \mathrm{mg} / \mathrm{g}$ rock [13]. Most of the adsorption that occurs in Berea sandstone is due to the few percentage of kaolinite clay that is within this sandstone.

The composition provided by the supplier for the kaolinite has the following major components (weight percents): $\mathrm{SiO}_{2} 44.2 \%, \mathrm{AlO}_{3} 39.7 \%, \mathrm{TiO}_{2} 1.39 \%, \mathrm{FeO}_{3}$ $0.13 \%$ with trace amounts of sodium, manganese, calcium, potassium, phosphorous, and fluorine. The specific surface area of the kaolinite is approximately $10 \mathrm{~m}^{2} / \mathrm{g}$. 
After the 12-hour exposure period, the sample is centrifuged and the supernatant was analyzed for residual surfactant concentration via UV-spectrophotometer. Knowing the absorbance value of the starting surfactant material, one can calculate the mass of surfactant that is left in the supernatant solution after measuring the absorbance of the supernatant.

\subsection{Water Flooding}

The value for initial water saturation $\left(\mathrm{S}_{\mathrm{wi}}\right)$ for this core is $26 \%$. Then the Berea core was saturated with brine under vacuum and then brought to connate water condition by injecting octane. The core was then subjected to water flood at a rate of $0.06 \mathrm{~mL} / \mathrm{min}$ until it reached the residual oil saturation.

\subsection{Surfactant Flooding}

The best surfactant and cosolvent formulation (Polysorbate20/1-octanol) which having the lowest IFT was injected onto Berea core at a rate of $0.06 \mathrm{~mL} / \mathrm{min}$. This is approximately a frontal advance rate of $0.5 \mathrm{ft} /$ day. Incremental oil recoveries were measured against time. All steps occurred at room temperature and ambient pressure.

\section{RESULTS AND DISCUSSION}

\subsection{Interfacial Tension Versus Surfactant-Cosolvent Type}

Different types of co-solvents alcohol being evaluated included several $\mathrm{n}$-alcohols ranging from $\mathrm{C} 3$ to $\mathrm{C} 10$. The aqueous phase had $1 \mathrm{wt} \%$ cosolvent concentration and had a default brine salinity of $2 \mathrm{wt} \% \mathrm{NaCl}$. The oil and aqueous surfactant solutions were mixed at a 1:1 volume ratio and equilibrated at ambient temperature. Fig. (2) shows IFT results with the different surfactant concentration with noctane at $25^{\circ} \mathrm{C}$.

Note that the IFT for Polysorbate20 alone was about 0.06 dyne/cm, and for an alcohol alone the IFT was over several dynes/cm, even greater than 30 dynes/cm $[14,15]$. One explanation for the synergistic action of the added alcohols is that they pack at the interface so as to decrease the curvature of the interfacial layer and thereby reducing the IFT. Perhaps the notion of a 'hydrophobic linker' is a good physical model for the action of these co-surfactants [16]. That is, an additive may work by linking the oil and surfactant molecules better at the interface. General observation is that all alcohol cosolvent act to decrease the IFT of the main surfactant.

There is an 'optimal' alcohol co-surfactant that creates the lowest IFT condition, less than $0.01 \mathrm{dyne} / \mathrm{cm}$. The IFT behavior versus the amount of Polysorbate20 and n-alcohol are fairly constant. This suggests that the desirable result that the low IFT condition may be attained with low concentrations of Polysorbate20 surfactant.

\subsection{Interfacial Tension Versus Temperature}

Measured IFT results in this study confirm that Polysorbate20 formulations may be largely indifferent to changes in the temperature as shown in Fig. (3). This is desirable because in oil reservoirs, the temperature will vary from zone to zone, with higher temperatures occurring in deeper subsurface depths $[10,17,18]$. This behavior means that one may formulate just a single aqueous based surfactant that is able to mobilize the crude oil as efficiently in spite of these temperature differences.

\subsection{Interfacial Tension Versus Salinity}

The salinity in the brine in the subsurface oil reservoir of course may vary both in an areal and vertical extent. Mature fields that have been subjected to years of waterflood often have substantial differences in salinity, for example, due to contrasts between the injected and original formation brine.

The measured IFT for this study versus the salinity as shown in Fig. (4) testifies no big difference in salinity gradient. Having a single surfactant formulation that is indifferent to salinity should be an advantage in the EOR design. With Polysorbate20-cosolvent formulations, one may accomplish the same objectives of having low IFT and avoiding surfactant phase trapping by changing the ratio of the surfactant formulation [19]. In addition, there is the possibility of formulating a low IFT for reservoirs containing high salinity.

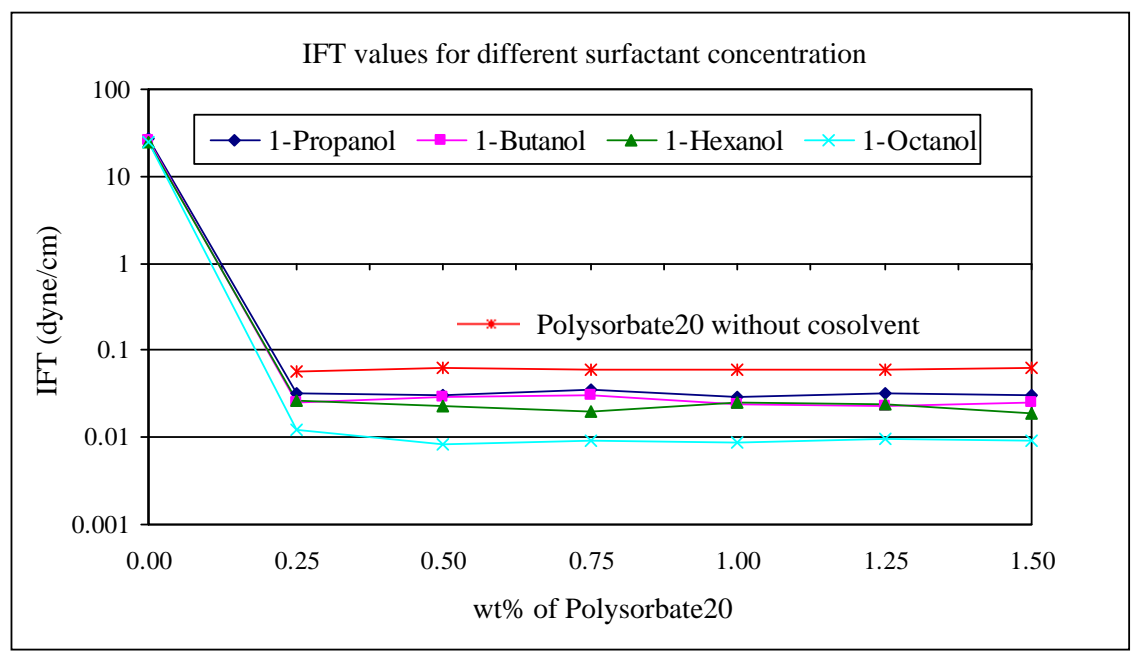

Fig. (2). IFT measured for equilibrated samples containing Polysorbate 20 mixed with small $n$-alcohol formulations versus noctane. 


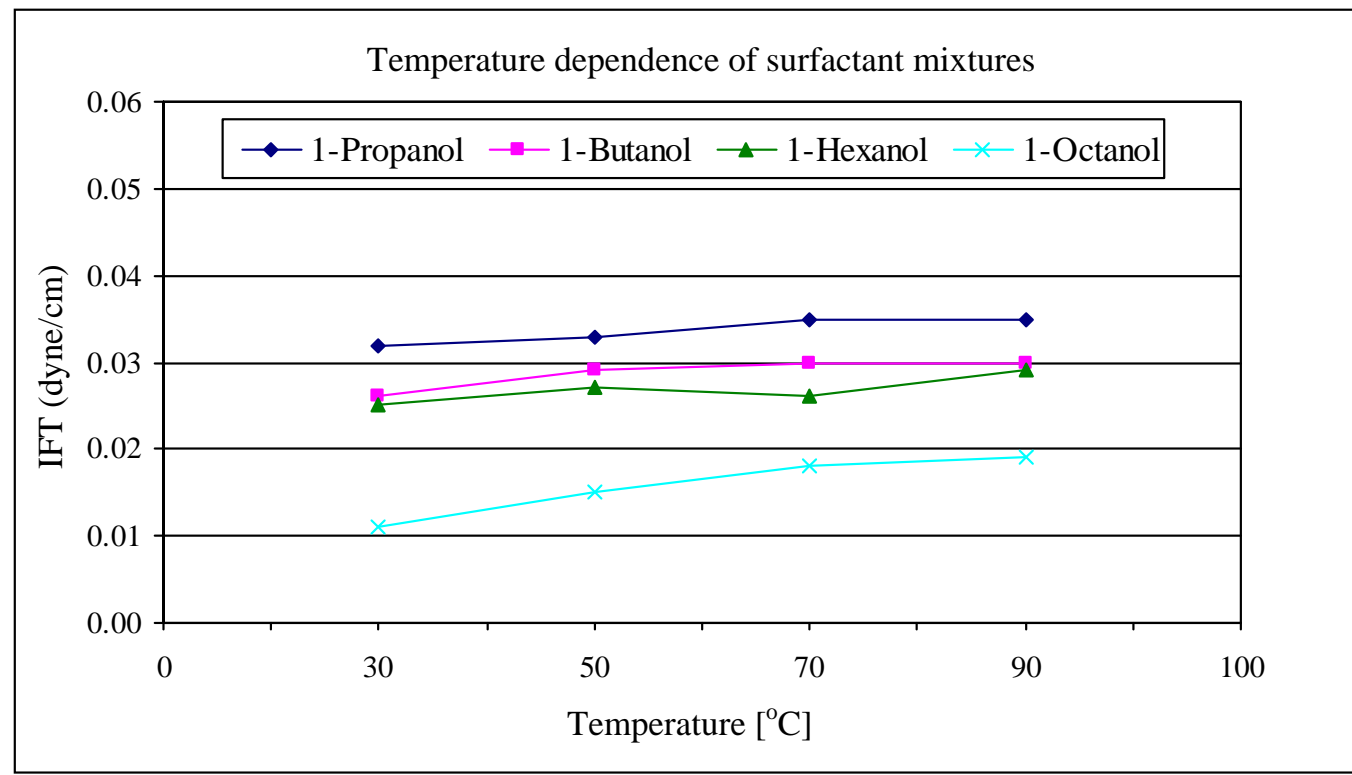

Fig. (3). IFT is nearly independent of temperature for Polysorbate20 versus n-octane

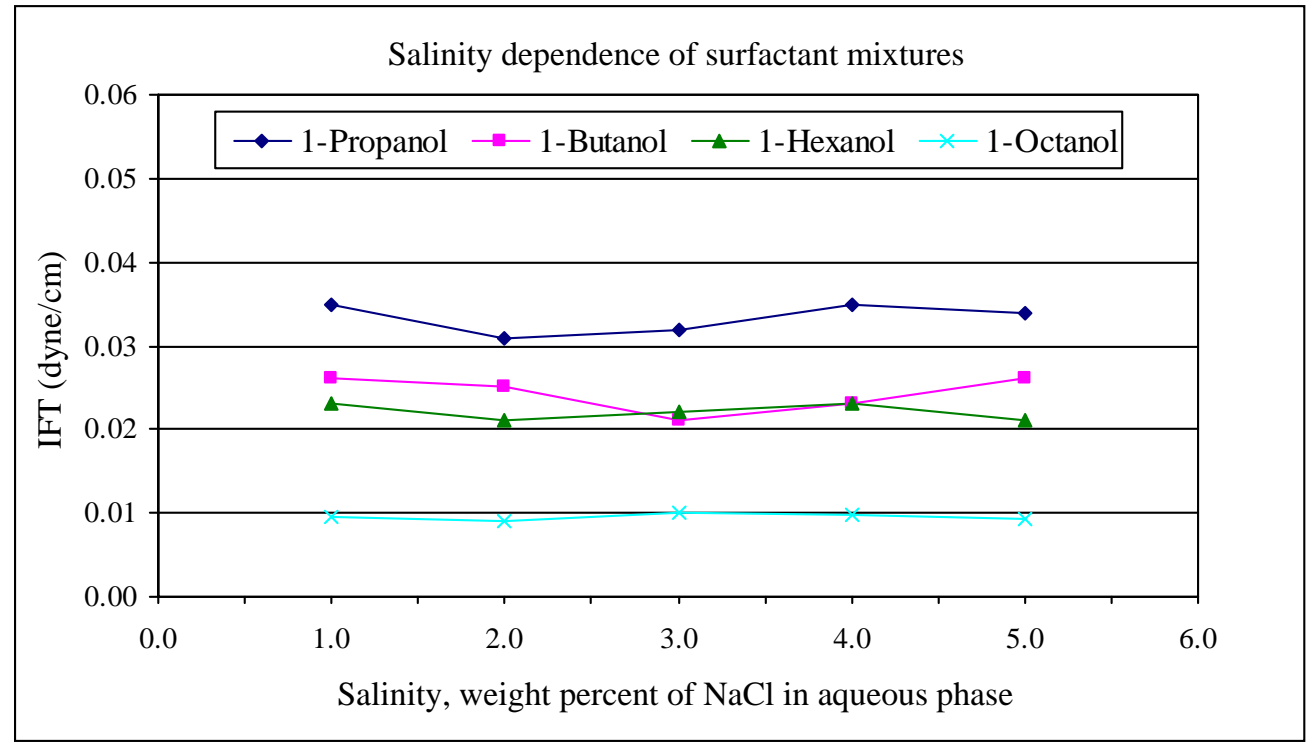

Fig. (4). IFT is nearly independent of salinity for Polysorbate20 versus n-octane.

\subsection{Surfactant Adsorption}

Maximum adsorptions measured for the different formulation of surfactants are shown in Table 2. Tests were conducted in $2 \mathrm{wt} \%$ of $\mathrm{NaCI}$ with a weight ratio of solution to solid of 20:1. Some trends evident from these data; the adsorption levels with mixtures of different n-alcohols are almost independent of the specific alcohol selected.

The surfactant adsorption on the crushed sandstone was significantly lower as expected because the clay content would be only a few percent. In general, the level of surfactant adsorption on kaolinite and crushed sandstone are within the normal range compared to commercially available surfactant used for EOR.

\subsection{Water Flood Oil Recovery}

Initially the core was flooded with octane until the core reach initial water saturation. At this stage, original oil in place was $74 \%$ which is $119 \mathrm{~mL}$ then the core was flooded with synthetic formation brine until less than $5 \%$ oil cut was being produced. Pump rate was adjusted so that the pressure gradient remained on the order of 1-2 psi/ft. At the end of water flooding, end point oil saturation was calculated. Fig. (5) shows the oil recovery obtained with the remaining oil saturation of $32 \%$.

\subsection{Surfactant Flood Oil Recovery}

The selected surfactant-cosolvent combination (1 wt $\%$ Polysorbate $20+1 \mathrm{wt} \%$ 1-octanol) was injected at a low flow rate at a particular pressure remained, based on the experience during the waterflooding to keep pressure gradient low. Effluent was collected in a fraction collector of $6 \mathrm{~mL}$ increment in graduated test tubes. When the surfactant breakthrough was observed, increments were reduced to 3 $\mathrm{mL}$ so that the surfactant concentration could be measured by titration on smaller samples to give a better estimate of the surfactant retention. $1 \mathrm{wt} \%$ of Polysorbate 20 was chosen instead of $0.25 \mathrm{wt} \%$ as can be seen in Fig. (2) to make sure 
Table 2. Maximum Adsorption Value of Surfactant Formulation

\begin{tabular}{|l|c|c|}
\hline Surfactant mixture & Kaolinite & Crushed sandstone \\
\hline \hline Polysorbate20 + 1-Propanol & $15.325 \mathrm{mg} / \mathrm{g}$ & $0.095 \mathrm{mg} / \mathrm{g}$ \\
\hline Polysorbate20 + 1-Butanol & $17.621 \mathrm{mg} / \mathrm{g}$ & $0.105 \mathrm{mg} / \mathrm{g}$ \\
\hline Polysorbate20 + 1-Hexanol & $16.862 \mathrm{mg} / \mathrm{g}$ & $0.112 \mathrm{mg} / \mathrm{g}$ \\
\hline Polysorbate20 + 1-Octanol & $15.742 \mathrm{mg} / \mathrm{g}$ & $0.098 \mathrm{mg} / \mathrm{g}$ \\
\hline
\end{tabular}

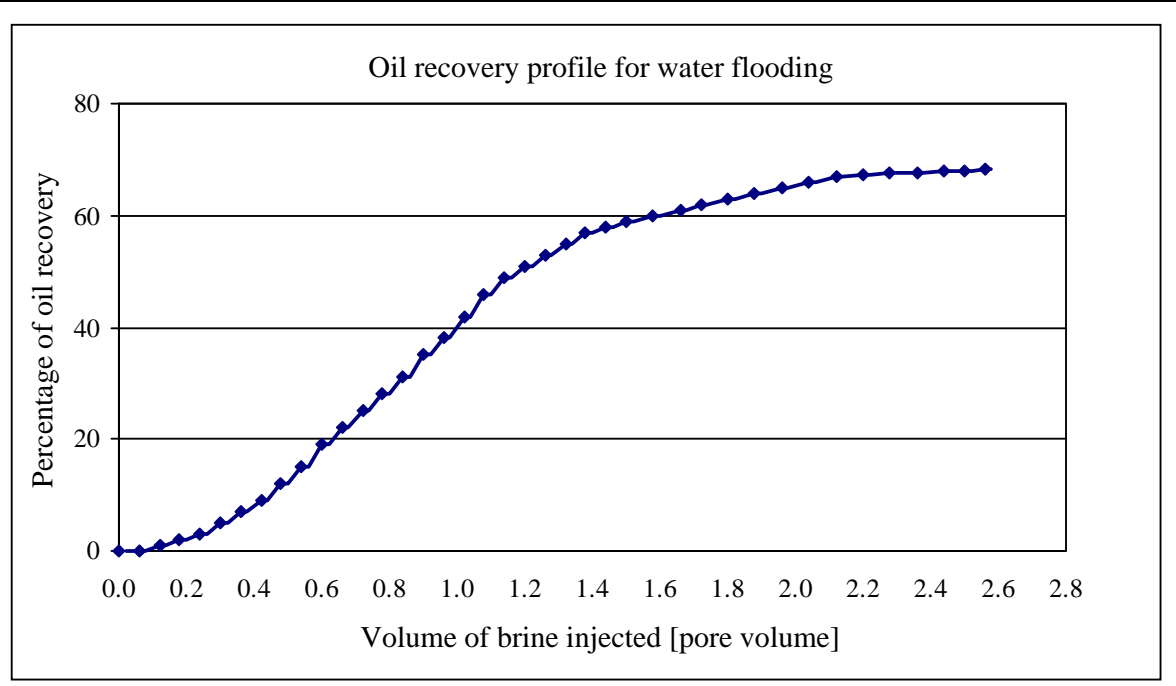

Fig. (5). Secondary oil recovery observed by water flooding.

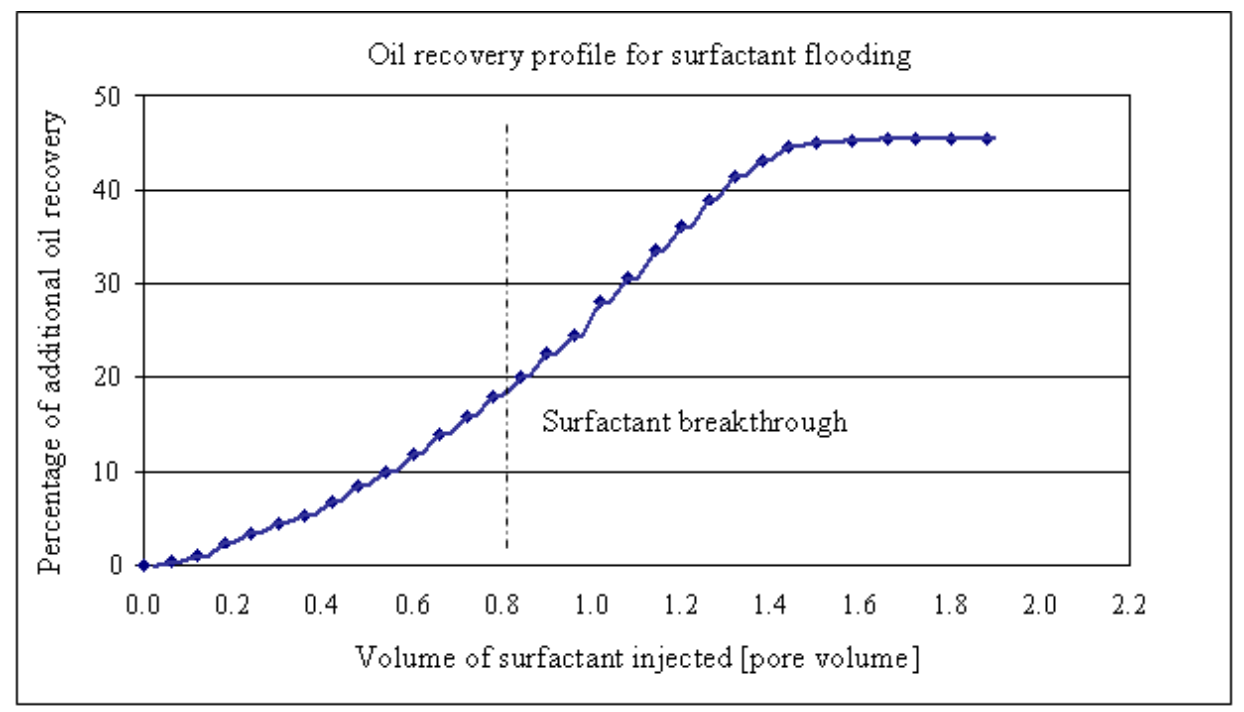

Fig. (6). Tertiary oil recovery observed by surfactant flooding.

the surfactant can perform well after considering the adsorption effect.

The results of the coreflood are displayed in Fig. (6). This formulation showed a good tertiary oil recovery which almost $46 \%$ of waterflood residual oil was recovered, reducing oil saturation from $32 \%$ to $17.3 \%$ even at high salinity (refer to Fig. 4). This formulation could be attractive as many reservoirs in the world have formation brine that has several percentages or more concentration of dissolved salts. In summary, this formulation was efficient in terms of recovering additional oil out of cores at waterflood residual oil saturation.

\section{CONCLUSIONS}

Key findings from this study include: 
1) Polysorbate20 surfactant may be formulated in brine solution that can create low IFT approaching 0.01 dyne/cm, or less, versus simple alkane hydrocarbon.

2) Polysorbate20 formulation may generate a low IFT that is largely independent of both salinity and temperature effects.

3) Polysorbate20 formulation exhibit relatively low adsorption on kaolinite clay and crushed sandstone.

4) This formulation was successful in terms of producing significant amounts of additional incremental oil (after water flooding).

\section{CONFLICT OF INTEREST}

Declared none.

\section{ACKNOWLEDGEMENTS}

The authors wish to thank the Department of Chemical and Biochemical, Dongguk University for their support in this project.

\section{NOMENCLATURE}

$\begin{array}{ll}\text { IFT } & =\text { Interfacial tension }[\mathrm{mN} / \mathrm{m}] \\ \text { EOR } & =\text { Enhanced oil recovery } \\ \mathrm{NaCl} & =\text { Sodium chloride } \\ \mathrm{wt} \% & =\text { Weight percent } \\ \mathrm{N}_{\text {cap }} & =\text { Capillary number } \\ \mathrm{v} & =\text { Darcy velocity }[\mathrm{m} / \mathrm{s}] \\ \mu & =\text { Viscosity of injected phase [Pa.s] } \\ \mathrm{rpm} & =\text { Revolution per minute }\end{array}$

\section{REFERENCES}

[1] W. W. Gale, and E. I. Sandvik, "Tertiary surfactant flooding: petroleum sulfonate composition efficacy studies", Soc. Pet. Eng. J., vol. 13, pp. 191-199, 1973.

[2] D. O. Shah, and R. S. Schechter, Improved Oil Recovery by Surfactant and Polymer Flooding, Academic Press: New York, 1977, pp. 205-252.

[3] K. Chunhee, and H. You-Lo, "Wetting and absorbency of nonionic surfactant solutions on cotton fabrics", Colloids Surf. A., vol. 187, pp. 385-397, 2001.
[4] K. Hill, and O. Rhode, "Sugar-based surfactants for consumer products and technical applications", Fett/Lipid, vol. 10, pp. 25-33, 1999.

[5] A. Abrams, "The influence of fluid viscosity, interfacial tension, and flow velocity on residual oil saturation left by waterflood", Soc.Pet.Eng. J., vol. 15, pp. 437-447, 1975.

[6] J. L. Cayias, R. S. Schechter, and W. H. Wade, "Modeling Crude Oils for Low Interfacial Tension”, Paper SPE 5813. Proceedings of the SPE Symposium on Improved Oil Recovery. March 22-24, 1976.

[7] E. M. Kutschmann, G. H. Findenegg, D. Nickel, and W. Rybinski, "Interfacial tension of alkylglucosides in different APG/oil/water systems", Colloid Polym. Sci., vol. 273, pp. 565 -571, 1995.

[8] D. A. Sabatini, E. Acosta, and J. H. Harwell, "Linker molecules in surfactant mixtures", Curr. Opin. Colloid Interface Sci., vol. 8, no. 4, pp. 316-326, 2003.

[9] S. Iglauer, and X. Zhang, "Lower Cost Methods for Improved Oil Recovery (IOR) via Surfactant Flooding", DOE Project DE-FC 2601BC15362, Final Report, California Institute of Technology, 2004.

[10] J. Novosad, "Surfactant retention in berea sandstone - effects of phase behavior and temperature", Soc.Pet.Eng. J., vol. 22, pp. 962970, 1982.

[11] A. K. Flaaten, Q. P. Nguyen, G. A. Pope, and J. Zhang, "A systematic laboratory approach to low-cost, high-performance chemical flooding", SPE Reserv. Eval. Eng., vol. 12, pp. 713-723, 2009.

[12] Y. Barakat, S. A. El-Mergawy, S. M. El-Zein, and A. I. Mead, "Adsorption of alkylbenzene sulfonates onto mineral surfaces", Indian J. Chem. Technol., vol. 2, no. 3, pp. 162-166, 1995.

[13] H. S. Hanna, and P. Somasundaran, "Equilibration of kaolinite in aqueous inorganic and surfactant solutions", J. Colloid Interface Sci., vol. 70, no. 1, pp. 181-191, 1979.

[14] P. H. Doe, W. H. Wade, and R. S. Schechter, "Alkyl benzene sulfonates for producing low interfacial tensions between hydrocarbons and water", J. Colloid Interface Sci., vol. 59, no. 3, pp. 525-531, 1977.

[15] M. Kahlweit, R. Strey, and G. Busse, "Effect of alcohols on the phase behavior of microemulsions", J. Phys. Chem., vol. 95, no. 13, pp. 5344-5352, 1991.

[16] A. Edgar, U. Hirotaka, A. S. David, and H. H. Jeffrey, "The role of hydrophilic linkers", J. Surfactants Deterg., vol. 5, no. 2, pp. 151157, 2002.

[17] P. Berger, and C. H. Lee, Ultra-low Concentration Surfactants for Sandstone and Limestone Floods, Paper SPE 75186. Proceedings of the SPE/DOE Symposium on Improved Oil Recovery, Tulsa. 2002.

[18] J. L. Cayias, R. S. Schechter, and W. H. Wade, "The utilization of petroleum sulfonates for producing low interfacial tensions between hydrocarbons and water", J. Colloid Interface Sci., vol. 59, no. 1, pp. 31-38, 1977.

[19] T. Förster, B. Guckenbiehl, H. Hensen, and W. Von Rybinski, "Physico-chemical basics of micro emulsions with alkyl polyglycosides", Prog. Colloid Polym. Sci., vol. 101, pp. 105-112, 1996. 